\title{
STABILITY OF JIN-XIN RELAXATION SHOCKS
}

\author{
BY \\ JEFFREY HUMPHERYS \\ Department of Mathematics, The Ohio State University, Columbus, Ohio 43210
}

\begin{abstract}
We examine the spectrum of shock profiles for the Jin-Xin relaxation scheme for systems of hyperbolic conservation laws in one spatial dimension. By using a weighted norm estimate, we prove that these shock profiles exhibit strong spectral stability in the weak shock limit.
\end{abstract}

1. Introduction. In this paper, we investigate the stability of shock profiles for the Jin-Xin model [JX]

$$
\left(\begin{array}{l}
U \\
V
\end{array}\right)_{t}+\left(\begin{array}{ll}
0 & I \\
A & 0
\end{array}\right)\left(\begin{array}{l}
U \\
V
\end{array}\right)_{x}=\left(\begin{array}{c}
0 \\
f(U)-V
\end{array}\right),
$$

where $f, U, V \in \mathbb{R}^{N}, f \in C^{3}, x \in \mathbb{R}$, and $A \in \mathbb{R}^{N \times N}$ is constant. This system falls into the general class of relaxation systems,

$$
W_{t}+F(W) W_{x}=Q(W)
$$

and serves as the basis for an important numerical scheme for approximating solutions of hyperbolic conservation laws

$$
U_{t}+f(U)_{x}=0 .
$$

It is easily verified that traveling wave solutions of (1) correspond to those for the viscous conservation laws

$$
U_{t}+f(U)_{x}=\left(B(U) U_{x}\right)_{x}
$$

where $B(U)=A-s^{2} I$; see Sec. 2 below.

The stability of viscous conservation laws is an area of great interest. Recently it has been shown that under suitable conditions, viscous shocks (3) are orbitally stable if and only if they exhibit strong spectral stability $[\mathrm{ZH}]$. This implies that orbital stability ultimately rests on the character of the spectrum, as is the case for classical dynamical systems. More recently yet, Mascia and Zumbrun [MZ] have extended this analysis to

Received December 6, 2000.

2000 Mathematics Subject Classification. Primary 35L65.

Key words and phrases. Jin-Xin relaxation model, conservation laws, stability of shock waves.

This research was completed at Indiana University in the course of the author's doctoral study. Special thanks to the author's thesis advisor, Kevin Zumbrun, for his steadfast guidance and encouragement throughout this work.

E-mail address: jeffh@math.ohio-state.edu 
Jin-Xin relaxation shocks, hence showing that strong spectral stability for Jin-Xin shocks is a sufficient condition for orbital stability.

In Jin and Xin's original work [JX], they showed this system to have an $L^{1}$ contraction property for scalar shocks $(N=1)$, which implies orbital stability. By using energy methods, we also show that the scalar case is stable. However, a gencralization of our method to a weighted norm estimate extends our scalar result to higher dimensions for weak shocks. H. Liu [L] recently proved orbital stability under zero-mass perturbations, a result slightly more general than this one. However, in light of Mascia and Zumbrun's recent work, one can get from spectral stability to orbital stability directly, and so much of Liu's analysis can be avoided. There is a close similarity between our approach and that of Goodman $[G]$ for the linear stability of weak shocks in dissipative conservation laws, under zero-mass perturbations.

Recently, Godillon [Go] carried out stability index calculations for the Jin-Xin model, which are consistent with stability for weak shocks. While this is an encouraging result, consistency only serves as a necessary condition for stability. Since our stability results only hold generally in the weak limit, other techniques will need to be explored to expand these results to stronger shocks, e.g., numerical Evans function calculations [B].

\section{Preliminaries.}

2.1. Assumptions. We assume $D f$ is strictly hyperbolic at a base point $\left(U_{0} . V_{0}\right)$. Thus, there exists $L, R$, such that

$$
L \cdot D f\left(U_{0}, V_{0}\right) \cdot R=\Lambda=\operatorname{diag}\left(\Lambda_{1}, \Lambda_{2}, \ldots, \Lambda_{N}\right)
$$

Moreover, we assume that $L, R$ also diagonalize $A$, and satisfy

$$
L \cdot\left(A-D f\left(U_{0}, V_{0}\right)^{2}\right) \cdot R>0 .
$$

In addition, we assume that $D f$ is genuinely nonlinear, that is, without loss of generality that

$$
\nabla \Lambda_{1} \cdot \vec{r}_{1}>0
$$

at $\left(U_{0}, V_{0}\right)$, where $\vec{r}_{1}$ is the right eigenvector of $D f$ corresponding to $\Lambda_{1}$. Note that no ordering in magnitude is assumed on $\Lambda_{j}$.

We remark that assumption (5) seems overly restrictive; however, we show in Sec. 5 that this is equivalent to symmetrizability of system (1). Moreover, we note that simultaneous diagonalizability of $D f$ and $A$ is more general than the usual assumption that $A=a I$, see [JX]. However, we also show in Sec. 5 that the usual assumption is actually sharp for most choices of $f$.

2.2. Shock profile. By a Jin-Xin shock profile, we mean a traveling wave solution of (1)

$$
\begin{aligned}
& U(x, t)=\widehat{U}(x-s t), \\
& V(x, t)=\widehat{V}(x-s t),
\end{aligned}
$$


with an asymptotically constant boundary $(\widehat{U}( \pm \infty), \widehat{V}( \pm \infty))=\left(U_{ \pm}, V_{ \pm}\right)$. By translating $x \rightarrow x-s t$, we can instead consider stationary solutions of

$$
\left(\begin{array}{l}
U \\
V
\end{array}\right)_{t}+\left(\begin{array}{cc}
-s I & I \\
A & -s I
\end{array}\right)\left(\begin{array}{l}
U \\
V
\end{array}\right)_{x}=\left(\begin{array}{c}
0 \\
f(U)-V
\end{array}\right)
$$

Thus, the shock profiles are solutions of the ordinary differential equation

$$
\begin{aligned}
-s U^{\prime}+V^{\prime} & =0, \\
-s V^{\prime}+A U^{\prime} & =f(U)-V,
\end{aligned}
$$

with limiting boundary values $U( \pm \infty)=U_{ \pm}$and $V( \pm \infty)=V_{ \pm}$. By combining and integrating (8) we arrive at

$$
U^{\prime}=\left(A-s^{2} I\right)^{-1}\left(f(U)-s\left(U-U_{-}\right)-V_{-}\right) .
$$

Since $U^{\prime}( \pm \infty)=0$, we have that $f\left(U_{-}\right)=V_{-}$. Finally, we write the profile as $\widehat{U}$, satisfying

$$
\widehat{U}^{\prime}=\left(A-s^{2} I\right)^{-1}\left(f(\widehat{U})-f\left(U_{-}\right)-s\left(\widehat{U}-U_{-}\right)\right) .
$$

Notice that this is exactly the same profile equation that comes from viscous shock profiles of (3), satisfying $U( \pm \infty)=U_{ \pm}$, where $B=A-s^{2} I$ :

$$
\widehat{U}^{\prime}=B(U)^{-1}\left(f(\widehat{U})-f\left(U_{-}\right)-s\left(\widehat{U}-U_{-}\right)\right) .
$$

This observation allows us to apply the weak shock analysis of Majda and Pego [MP] for viscous shocks to Jin-Xin shocks; see Sec. 4.

2.3. Spectral Stability. We say that a shock profile has strong spectral stability if the linearized operator $L$ (about the shock profile) has no spectrum in the closed right deleted half-plane, $D=\{\mathcal{R} e(\lambda) \geq 0\} \backslash\{0\}$, i.e., no growth or oscillatory modes. One can readily show that no essential spectrum for Jin-Xin shocks exists in $D$ by considering the essential spectrum of the constant states $U_{ \pm}$; see [Ze], [Z], [MZ]. Hence, to show strong spectral stability for the Jin-Xin system, it suffices to show that no eigenvalues (point spectrum) exist in $D$. We remark that traveling wave profiles always have a zero eigenvalue associated with its translational invariance.

By linearizing (7) about the profile $\widehat{U}$, we get the following eigenvalue problem:

$$
\lambda\left(\begin{array}{l}
U \\
V
\end{array}\right)+\left(\begin{array}{cc}
-s I & I \\
A & -s I
\end{array}\right)\left(\begin{array}{l}
U \\
V
\end{array}\right)_{x}=\left(\begin{array}{cc}
0 & 0 \\
D f(\widehat{U}) & -I
\end{array}\right)\left(\begin{array}{l}
U \\
V
\end{array}\right) .
$$

Suppose that $(U, V)$ is an eigenfunction of (11) with an eigenvalue $\lambda \in D$. Define

$$
\widetilde{U}(x)=\int_{-\infty}^{x} U(z) d z .
$$

Then by integrating the top equation of (11), from $\infty$ to $-\infty$, we get that $\widetilde{U}$ and its derivatives decay exponentially as $x \rightarrow \infty$. Thus, we can recast $(11)$ in terms of $(\tilde{U}, V)$ as (suppressing the tilde)

$$
\begin{aligned}
\lambda U-s U^{\prime}+V & =0, \\
\lambda V-s V^{\prime}+A U^{\prime \prime} & =D f(\widehat{U}) U^{\prime}-V .
\end{aligned}
$$


This is the integrated eigenvalue equation. Its point spectrum differs from that of (11) only at $\lambda=0$. Thus, to show that Jin-Xin shocks exhibit strong spectral stability, it suffices to show that (12) has only negative point spectra.

3. Scalar case. In this section we prove that the scalar, $N=1$, eigenvalue equation (11) exhibits strong spectral stability. The integrated coordinate eigenvalue problem (12) takes the form

$$
\begin{aligned}
\lambda u-s u^{\prime}+v & =0, \\
\lambda v-s v^{\prime}+A u^{\prime \prime} & =f^{\prime}(\hat{u}) u^{\prime}-v,
\end{aligned}
$$

where $u, v \in \mathbb{R}, \hat{u}$ is the profile, $\hat{u}_{x}<0, f^{\prime \prime}>0$, and $A>f^{\prime}(\hat{u})^{2}$.

Theorem 1. Scalar Jin-Xin shocks exhibit strong spectral stability.

Proof. It suffices to show that (13) has no spectrum with $\operatorname{Re}(\lambda) \geq 0$. We refer to Lemma 1 below for the following identities, which hold for $\mathcal{R} e(\lambda) \geq 0$ :

$$
\begin{aligned}
& \text { (i) } \int_{-\infty}^{+\infty}|v|^{2} \leq \int_{-\infty}^{+\infty}\left|f^{\prime}(\hat{u}) u^{\prime} \bar{v}\right| . \\
& \text { (ii) } \int_{-\infty}^{+\infty} A\left|u^{\prime}\right|^{2}<\int_{-\infty}^{+\infty}|v|^{2} .
\end{aligned}
$$

By adding half of (ii) to (i), we get

$$
\frac{1}{2} \int_{-\infty}^{+\infty}\left(|v|^{2}+A\left|u^{\prime}\right|^{2}\right)<\int_{-\infty}^{+\infty}\left|f^{\prime}(\hat{u}) u^{\prime} \bar{v}\right|
$$

which by Young's inequality yields

$$
\frac{1}{2} \int_{-\infty}^{+\infty}\left(|v|^{2}+A\left|u^{\prime}\right|^{2}\right)<\frac{1}{2} \int_{-\infty}^{+\infty}\left(|v|^{2}+f^{\prime}(\hat{u})^{2}\left|u^{\prime}\right|^{2}\right) .
$$

This is a contradiction since $A \geq f^{\prime}(\hat{u})^{2}$; see (5). Thus $\operatorname{Re}(\lambda)<0$.

Lemma 1. For $\operatorname{Re}(\lambda) \geq 0$, (i) and (ii) in the above proof hold.

Proof. (i) We begin by multiplying (13b) by the conjugate $\bar{v}$ and integrating from $-\infty$ to $\infty$. We get

$$
(\lambda+1) \int_{-\infty}^{+\infty}|v|^{2}-s \int_{-\infty}^{+\infty} v^{\prime} \bar{v}+\int_{-\infty}^{+\infty} A u^{\prime \prime} \bar{v}=\int_{-\infty}^{+\infty} f^{\prime}(\hat{u}) u^{\prime} \bar{v} .
$$

We take the real part and note that the second term vanishes, leaving us with

$$
(\mathcal{R} e(\lambda)+1) \int_{-\infty}^{+\infty}|v|^{2}+\mathcal{R} e\left(\int_{-\infty}^{+\infty} A u^{\prime \prime} \bar{v}\right)=\mathcal{R} e\left(\int_{-\infty}^{+\infty} f^{\prime}(\hat{u}) u^{\prime} \bar{v}\right) .
$$

Finally, by replacing $\bar{v}$ with $s \bar{u}^{\prime}-\bar{\lambda} \bar{u}$ from (13a) and appropriately integrating by parts, we arrive at

$$
\mathcal{R} e(\lambda) \int_{-\infty}^{+\infty}\left(|v|^{2}+A\left|u^{\prime}\right|^{2}\right)+\int_{-\infty}^{+\infty}|v|^{2}=\mathcal{R} e\left(\int_{-\infty}^{+\infty} f^{\prime}(\hat{u}) u^{\prime} \bar{v}\right) .
$$


Thus, for $\operatorname{Re}(\lambda) \geq 0$, we have

$$
\int_{-\infty}^{+\infty}|v|^{2} \leq \mathcal{R} e\left(\int_{-\infty}^{+\infty} f^{\prime}(\hat{u}) u^{\prime} \bar{v}\right) \leq \int_{-\infty}^{+\infty}\left|f^{\prime}(\hat{u}) u^{\prime} \bar{v}\right|
$$

(ii) We construct this identity by multiplying (13b) by the conjugate $\bar{u}$ and integrating from $-\infty$ to $\infty$. We get

$$
(\lambda+1) \int_{-\infty}^{+\infty} v \bar{u}-s \int_{-\infty}^{+\infty} v^{\prime} \bar{u}+\int_{-\infty}^{+\infty} A u^{\prime \prime} \bar{u}=\int_{-\infty}^{+\infty} f^{\prime}(\hat{u}) u^{\prime} \bar{u} .
$$

Integrating the second and third terms by parts and adjusting terms yields

$$
(\lambda+\bar{\lambda}+1) \int_{-\infty}^{+\infty} v \bar{u}-\int_{-\infty}^{+\infty} v\left(\bar{\lambda} \bar{u}-s \bar{u}^{\prime}\right)=\int_{-\infty}^{+\infty} A\left|u^{\prime}\right|^{2}+\int_{-\infty}^{+\infty} f^{\prime}(\hat{u}) u^{\prime} \bar{u}
$$

which gives

$$
(2 \mathcal{R} e(\lambda)+1) \int_{-\infty}^{+\infty} v \bar{u}+\int_{-\infty}^{+\infty}|v|^{2}=\int_{-\infty}^{+\infty} A\left|u^{\prime}\right|^{2}+\int_{-\infty}^{+\infty} f^{\prime}(\hat{u}) u^{\prime} \bar{u}
$$

Now, take the real part:

$$
\begin{aligned}
(2 \mathcal{R} e(\lambda)+1) \mathcal{R} e & \left(\int_{-\infty}^{+\infty} v \bar{u}\right)+\int_{-\infty}^{+\infty}|v|^{2} \\
& =\int_{-\infty}^{+\infty} A\left|u^{\prime}\right|^{2}-\frac{1}{2} \int_{-\infty}^{+\infty} f^{\prime \prime}(\hat{u}) \hat{u}_{x}|u|^{2}
\end{aligned}
$$

By using (13a), we observe that

$$
\lambda \int_{-\infty}^{+\infty}|u|^{2}-s \int_{-\infty}^{+\infty} u^{\prime} \bar{u}+\int_{-\infty}^{+\infty} v \bar{u}=0
$$

Hence, by taking the real part, we have

$$
\mathcal{R} e\left(\int_{-\infty}^{+\infty} v \bar{u}\right)=-\mathcal{R} e(\lambda) \int_{-\infty}^{+\infty}|u|^{2}
$$

which goes into (14) to give

$$
\begin{aligned}
\int_{-\infty}^{+\infty}|v|^{2}=\mathcal{R} & (\lambda)((2 \mathcal{R} e(\lambda)+1)) \int_{-\infty}^{+\infty}|u|^{2} \\
& +\int_{-\infty}^{+\infty} A\left|u^{\prime}\right|^{2}-\frac{1}{2} \int_{-\infty}^{+\infty} f^{\prime \prime}(\hat{u}) \hat{u}_{x}|u|^{2}
\end{aligned}
$$

Thus for $\mathcal{R} e(\lambda) \geq 0$, we have

$$
\int_{-\infty}^{+\infty} A\left|u^{\prime}\right|^{2}<\int_{-\infty}^{+\infty}|v|^{2}
$$


4. System case. In this section we show that the eigenvalue problem (11) exhibits strong spectral stability for weak shocks. From (12), we have

$$
\begin{aligned}
\lambda U-s U^{\prime}+V & =0, \\
\lambda V-s V^{\prime}+A U^{\prime \prime} & =\operatorname{Df}(\widehat{U}) U^{\prime}-V .
\end{aligned}
$$

Recall from (4) and (5) that $L$ and $R$ diagonalize $D f\left(U_{0}, V_{0}\right)$, and thus there exist a $C^{2}$ choice of $R, L$ in a neighborhood of the base point $\left(U_{0}, V_{0}\right)$ satisfying both $L A R>\Lambda^{2}$ and $\widetilde{A}=L A R>\Lambda^{2}$. By transforming $U \rightarrow R U$ and $V \rightarrow R V$, we have

$$
\begin{aligned}
& \lambda R U-s\left(R U^{\prime}+R^{\prime} U\right)+R V=0 \\
& \lambda R V-s\left(R V^{\prime}+R^{\prime} V\right)+R \widetilde{A} L\left(R^{\prime \prime} U+2 R^{\prime} U^{\prime}+R U^{\prime \prime}\right) \\
& =R \Lambda L\left(R^{\prime} U+R U^{\prime}\right)-R V .
\end{aligned}
$$

Left multiplying by $L$ yiclds

$$
\begin{aligned}
& \lambda U-s\left(U^{\prime}+L R^{\prime} U\right)+V=0 \\
& \lambda V-s\left(V^{\prime}+L R^{\prime} V\right)+\widetilde{A}\left(L R^{\prime \prime} U+2 L R^{\prime} U^{\prime}+U^{\prime \prime}\right) \\
& \quad=\Lambda\left(L R^{\prime} U+U^{\prime}\right)-V .
\end{aligned}
$$

We apply to this the following two observations. First, following the analysis of Goodman $[\mathrm{G}]$ (see also $[\mathrm{Z}],[\mathrm{MZ}],[\mathrm{HuZ}]$ ), we can scale $L$ and $R$ so that

$$
\left(L R^{\prime}\right)_{11}=0
$$

And secondly, since both Jin-Xin shocks and viscous shocks satisfy the same profile equations, (9) and (10), the asymptotic analysis of Majda and Pego [MP] holds for JinXin shocks as well. Thus, we have the following lemma:

Lemma 2. In the weak shock limit, where $\epsilon=\left|u_{+}-u_{-}\right|$, we have $\left|\widehat{U}_{x}\right|=O\left(\epsilon^{2}\right)$ and $\left|\widehat{U}_{x x}\right|=O\left(\epsilon\left|\hat{u}_{x}\right|\right)$, for Jin-Xin shocks profiles. Moreover, under the genuine nonlinearity condition, $\Lambda_{1}^{\prime}<-\theta\left|\widehat{U}_{x}\right|$ for some $\theta>0$. Strict hyperbolicity implies without loss of generality that $\Lambda_{j}-s \neq 0$, for $j>1$.

Theorem 2. Weak Jin-Xin shocks, (11) exhibit strong spectral stability.

Proof. It suffices to show that (12) has no point spectrum with $\mathcal{R} e(\lambda) \geq 0$. We refer to Lemma 3 for the following identities, which hold in the weak shock limit for $\operatorname{Re}(\lambda) \geq 0$, 
where $u_{j}, v_{j}$ denote coordinates of $(U, V)$ in (15) and $L, R$ chosen as in (4):

(i) $\sum_{j=1}^{N} \int_{-\infty}^{+\infty} \alpha_{j}\left|v_{j}\right|^{2} \leq \sum_{j=1}^{N} \int_{-\infty}^{+\infty}\left|\alpha_{j} \Lambda_{j}\left\|v_{j}\right\| u_{j}^{\prime}\right|$

$$
+C_{1} \int_{-\infty}^{+\infty}\left|\widehat{U}_{x}\right|\left[\epsilon_{1}\left|u_{1}\right|^{2}+\sum_{j \neq 1}\left|u_{j}\right|^{2}+\sum_{j=1}^{N}\left(\left|v_{j}\right|^{2}+\left|u_{j}^{\prime}\right|^{2}\right)\right],
$$

(ii) $\sum_{j=1}^{N} \int_{-\infty}^{+\infty} \alpha_{j} \widetilde{A}_{j}\left|u_{j}^{\prime}\right|^{2}+\frac{1}{2} \sum_{j=1}^{N} \int_{-\infty}^{+\infty}\left(s \alpha_{j}^{\prime}-\left(\alpha_{j} \Lambda_{j}\right)^{\prime}\right)\left|u_{j}\right|^{2}$

$$
\leq \sum_{j=1}^{N} \int_{-\infty}^{+\infty} \alpha_{j}\left|v_{j}\right|^{2}+C_{2} \int_{-\infty}^{+\infty}\left|\widehat{U}_{x}\right|\left[\epsilon_{2}\left|u_{1}\right|^{2}+\frac{1}{\epsilon_{2}} \sum_{j \neq 1}\left|u_{j}\right|^{2}+\sum_{j=1}^{N}\left|u_{j}^{\prime}\right|^{2}\right]
$$

where $\alpha=\operatorname{diag}\left(\alpha_{1}, \alpha_{2}, \ldots, \alpha_{N}\right)$, is a positive-diagonal matrix satisfying $\alpha_{1}=1$ and for $j>1$,

$$
\begin{aligned}
\alpha_{j}^{\prime}(x) & =\frac{-C_{3}}{\Lambda_{j}-s}\left|\widehat{U}_{x}\right| \alpha_{j}(x), \\
\alpha_{j}(0) & =1
\end{aligned}
$$

Just as with the scalar case, we add half of (ii) to (i) and simplify to get

$$
\begin{gathered}
\frac{1}{2} \sum_{j=1}^{N} \int_{-\infty}^{+\infty} \alpha_{j}\left(\left|v_{j}\right|^{2}+\widetilde{A}_{j}\left|u_{j}\right|^{2}\right)+\frac{1}{2} \sum_{j=1}^{N} \int_{-\infty}^{+\infty}\left(s \alpha_{j}^{\prime}-\left(\alpha_{j} \Lambda_{j}\right)^{\prime}\right)\left|u_{j}\right|^{2} \\
\leq C_{4} \int_{-\infty}^{+\infty}\left|\widehat{U}_{x}\right|\left[\epsilon_{3}\left|u_{1}\right|^{2}+\frac{1}{\epsilon_{3}} \sum_{j \neq 1}\left|u_{j}\right|^{2}+\sum_{j=1}^{N}\left(\left|v_{j}\right|^{2}+\left|u_{j}^{\prime}\right|^{2}\right)\right] \\
\quad+\sum_{j=1}^{N} \int_{-\infty}^{+\infty}\left|\alpha_{j} \Lambda_{j}\left\|v_{j}\right\| u_{j}^{\prime}\right| .
\end{gathered}
$$

We claim that for $C_{3}$ sufficiently large and $\epsilon_{3},\left|\widehat{U}_{x}\right|$ sufficiently small,

$$
\frac{1}{2} \sum_{j=1}^{N} \int_{-\infty}^{+\infty}\left(s \alpha_{j}^{\prime}-\left(\alpha_{j} \Lambda_{j}\right)^{\prime}\right)\left|u_{j}\right|^{2} \geq C_{4} \int_{-\infty}^{+\infty}\left|\widehat{U}_{x}\right|\left[\epsilon_{3}\left|u_{1}\right|^{2}+\frac{1}{\epsilon_{3}} \sum_{j \neq 1}\left|u_{j}\right|^{2}\right]
$$

For $j=1$ and sufficiently small $\epsilon_{3}$, we have

$$
-\frac{1}{2} \int_{-\infty}^{+\infty} \Lambda_{1}^{\prime}\left|u_{1}\right|^{2} \geq \epsilon_{3} C_{4} \int_{-\infty}^{+\infty}\left|\widehat{U}_{x} \| u_{1}\right|^{2}
$$


since $\Lambda_{1}^{\prime} \geq-\theta\left|\widehat{U}_{x}\right|$. For $j \neq 1$ and $C_{3}$ sufficiently large,

$$
\begin{aligned}
\frac{1}{2} \int_{-\infty}^{+\infty}\left(s \alpha_{j}^{\prime}-\left(\alpha_{j} \Lambda_{j}\right)^{\prime}\right)\left|u_{j}\right|^{2} & \left.=\frac{1}{2} \int_{-\infty}^{+\infty}\left(\left(s-\Lambda_{j}\right) \alpha_{j}^{\prime}-\alpha_{j} \Lambda_{j}^{\prime}\right)\right)\left|u_{j}\right|^{2} \\
& =\frac{1}{2} \int_{-\infty}^{+\infty}\left(C_{3}\left|\widehat{U}_{x}\right| \alpha_{j}-\alpha_{j} \Lambda_{j}^{\prime}\right)\left|u_{j}\right|^{2} \\
& \geq \frac{C_{4}}{\epsilon_{3}} \int_{-\infty}^{+\infty}\left|\widehat{U}_{x} \| u_{j}\right|^{2}
\end{aligned}
$$

Thus, (17) becomes

$$
\begin{aligned}
& \frac{1}{2} \sum_{j=1}^{N} \int_{-\infty}^{+\infty} \alpha_{j}\left(\left|v_{j}\right|^{2}+\widetilde{A}_{j}\left|u_{j}^{\prime}\right|^{2}\right) \leq C_{4} \sum_{j=1}^{N} \int_{-\infty}^{+\infty}\left|\widehat{U}_{x}\right|\left(\left|v_{j}\right|^{2}+\left|u_{j}^{\prime}\right|^{2}\right) \\
& \quad+\sum_{j=1}^{N} \int_{-\infty}^{+\infty}\left|\alpha_{j} \Lambda_{j}\left\|v_{j}\right\| u_{j}^{\prime}\right| .
\end{aligned}
$$

Now since $\widetilde{A}-\Lambda^{2}>0, \exists \eta>0$ such that $\widetilde{A}-(1+\eta) \Lambda^{2}>\eta I$. Thus, by Young's inequality,

$$
\begin{aligned}
& \frac{1}{2} \sum_{j=1}^{N} \int_{-\infty}^{+\infty} \alpha_{j}\left(\left|v_{j}\right|^{2}+\widetilde{A}_{j}\left|u_{j}^{\prime}\right|^{2}\right) \leq C_{4} \sum_{j=1}^{N} \int_{-\infty}^{+\infty}\left|\widehat{U}_{x}\right|\left(\left|v_{j}\right|^{2}+\left|u_{j}^{\prime}\right|^{2}\right) \\
& \quad+\frac{1}{2} \sum_{j=1}^{N} \int_{-\infty}^{+\infty} \alpha_{j}\left[\frac{1}{1+\eta}\left|v_{j}\right|^{2}+(1+\eta)\left|\Lambda_{j}\right|^{2}\left|u_{j}^{\prime}\right|^{2}\right]
\end{aligned}
$$

which simplifies to

$$
\frac{1}{2} \sum_{j=1}^{N} \int_{-\infty}^{+\infty} \alpha_{j}\left[\frac{\eta}{1+\eta}\left|v_{j}\right|^{2}+\eta\left|u_{j}^{\prime}\right|^{2}\right] \leq C_{4} \sum_{j=1}^{N} \int_{-\infty}^{+\infty}\left|\widehat{U}_{x}\right|\left(\left|v_{j}\right|^{2}+\left|u_{j}^{\prime}\right|^{2}\right) .
$$

However, since $\alpha_{j}=1+O(\epsilon)$, then in the weak shock limit,

$$
\frac{\eta}{1+\eta} \alpha_{j} \gg 2 C_{4} \int_{-\infty}^{+\infty}\left|\widehat{U}_{x}\right|=O(\epsilon)
$$

$\forall j$. This is a contradiction. Thus $\mathcal{R} e(\lambda)<0$.

Lemma 3. For $\mathcal{R} e(\lambda) \geq 0$, and $L, R$ chosen as in (16), (i) and (ii) in the above proof hold, for weak shocks.

Proof. (i) We begin by taking the $L^{2}$ inner product of (15b) with $\alpha V$ to get

$$
\begin{aligned}
& \left\langle\alpha V .(\lambda+1) V-s\left(V^{\prime}+L R^{\prime} V\right)+\widetilde{A}\left(L R^{\prime \prime} U+2 L R^{\prime} U^{\prime}+U^{\prime \prime}\right)\right\rangle \\
& \quad=\left\langle\alpha V, \Lambda\left(U^{\prime}+L R^{\prime} U\right)\right\rangle .
\end{aligned}
$$

This simplifies to

$$
\begin{aligned}
& (\lambda+1)\langle\alpha V, V\rangle-s\left\langle\alpha V, L R^{\prime} V\right\rangle-s\left\langle\alpha V, V^{\prime}\right\rangle \\
& \quad=\left\langle\alpha V,\left(\Lambda L R^{\prime}-\tilde{A} L R^{\prime \prime}\right) U\right\rangle+\left\langle\alpha V,\left(\Lambda-2 \widetilde{A} L R^{\prime}\right) U^{\prime}\right\rangle-\left\langle\alpha V, \widetilde{A} U^{\prime \prime}\right\rangle
\end{aligned}
$$


Integrating the last term by parts and simplifying gives

$$
\begin{aligned}
(\lambda+1) & \langle V, \alpha V\rangle-s\left\langle V, \alpha L R^{\prime} V\right\rangle-s\left\langle V, \alpha V^{\prime}\right\rangle \\
& =\left\langle V, \alpha\left(\Lambda L R^{\prime}-\widetilde{A} L R^{\prime \prime}\right) U\right\rangle+\left\langle V,\left(\alpha \Lambda-2 \alpha \widetilde{A} L R^{\prime}+(\alpha \widetilde{A})^{\prime}\right) U^{\prime}\right\rangle \\
& +\left\langle V^{\prime}, \alpha \widetilde{A} U^{\prime}\right\rangle .
\end{aligned}
$$

By writing $V^{\prime}$ in terms of $U$ and its derivatives from (15a), we have

$$
\begin{aligned}
(\lambda+1) & \langle V, \alpha V\rangle-s\left\langle V, \alpha L R^{\prime} V\right\rangle-s\left\langle V, \alpha V^{\prime}\right\rangle \\
= & \left\langle V, \alpha\left(\Lambda L R^{\prime}-\widetilde{A} L R^{\prime \prime}\right) U\right\rangle+\left\langle V,\left(\alpha \Lambda-2 \alpha \widetilde{A} L R^{\prime}+(\alpha \widetilde{A})^{\prime}\right) U^{\prime}\right\rangle \\
& +\left\langle s\left(\left(L R^{\prime}\right)^{\prime} U+L R^{\prime} U^{\prime}+U^{\prime \prime}\right)-\lambda U^{\prime}, \alpha \widetilde{A} U^{\prime}\right\rangle .
\end{aligned}
$$

Take the real part:

$$
\begin{aligned}
& \sum_{j=1}^{N} \int_{-\infty}^{+\infty}\left[(\mathcal{R} e(\lambda)+1) \alpha_{j}\left|v_{j}\right|^{2}+\mathcal{R} e(\lambda) \alpha_{j} A_{j}\left|u_{j}^{\prime}\right|^{2}\right] \\
&= s \mathcal{R} e\left\langle V, \alpha L R^{\prime} V\right\rangle+\mathcal{R} e\left\langle V, \alpha\left(\Lambda L R^{\prime}-\widetilde{A} L R^{\prime \prime}\right) U\right\rangle \\
&+\operatorname{Re} e\langle V, \alpha \Lambda U\rangle-\mathcal{R} e\left\langle V,\left(2 \alpha \widetilde{A} L R^{\prime}-(\alpha \widetilde{A})^{\prime}\right) U^{\prime}\right\rangle \\
&+s \mathcal{R} e\left\langle\left(L R^{\prime}\right)^{\prime} U+L R^{\prime} U^{\prime}, \alpha \widetilde{A} U^{\prime}\right\rangle-s \sum_{j=1}^{N} \int_{-\infty}^{+\infty} \alpha^{\prime}\left(\left|v_{j}\right|^{2}+\left|u_{j}^{\prime}\right|^{2}\right)
\end{aligned}
$$

Note that in the small shock limit, $a^{\prime}, L R^{\prime}=O\left(\left|\widehat{U}_{x}\right|\right)$ and $L R^{\prime \prime}=O\left(\left|\widehat{U}_{x x}\right|+\left|\widehat{U}_{x}\right|^{2}\right)$. Thus for $\mathcal{R} e(\lambda) \geq 0$, we have

$$
\begin{aligned}
& \sum_{j=1}^{N} \int_{-\infty}^{+\infty} \alpha_{j}\left|v_{j}\right|^{2} \leq \sum_{j=1}^{N} \int_{-\infty}^{+\infty}\left|\alpha_{j} \Lambda_{j}\left\|v_{j}\right\| u_{j}^{\prime}\right| \\
& \quad+\sum_{j=1}^{N} \int_{-\infty}^{+\infty} O\left(\left|\widehat{U}_{x}\right|\right)\left(\left|v_{j}\right|^{2}+\left|u_{j}^{\prime}\right|^{2}\right)+\sum_{i, j} \int_{-\infty}^{+\infty} O\left(\left|\widehat{U}_{x}\right|\right)\left|v_{i} \| u_{j}\right| .
\end{aligned}
$$

Finally, by Young's inequality, we get

$$
\begin{aligned}
& \sum_{j=1}^{N} \int_{-\infty}^{+\infty} \alpha_{j}\left|v_{j}\right|^{2} \leq \sum_{j=1}^{N} \int_{-\infty}^{+\infty}\left|\alpha_{j} \Lambda_{j}\left\|v_{j}\right\| u_{j}^{\prime}\right| \\
& \quad+C_{1} \int_{-\infty}^{+\infty}\left|\widehat{U}_{x}\right|\left[\epsilon_{1}\left|u_{1}\right|^{2}+\sum_{j \neq 1}\left|u_{j}\right|^{2}+\sum_{j=1}^{N}\left(\left|v_{j}\right|^{2}+\left|u_{j}^{\prime}\right|^{2}\right)\right] .
\end{aligned}
$$

(ii) Now take the $L^{2}$ inner product of $(15 \mathrm{~b})$ with $\alpha U$. We get

$$
\begin{aligned}
& \left\langle\alpha U,(\lambda+1) V-s\left(V^{\prime}+L R^{\prime} V\right)+\widetilde{A}\left(L R^{\prime \prime} U+2 L R^{\prime} U^{\prime}+U^{\prime \prime}\right)\right\rangle \\
& \quad=\left\langle\alpha U, \Lambda\left(L R^{\prime} U+U^{\prime}\right)\right\rangle .
\end{aligned}
$$

Simplifying yields

$$
\begin{aligned}
& \left\langle\alpha U,(\lambda+1) V-s L R^{\prime} V\right\rangle+s\left\langle\alpha^{\prime} U, V\right\rangle+\left\langle s U^{\prime}, \alpha V\right\rangle \\
& \quad=\left\langle\alpha U,\left(\Lambda L R^{\prime}-\widetilde{A} L R^{\prime \prime}\right) U\right\rangle+\left\langle\alpha U,\left(\Lambda-2 \widetilde{A} L R^{\prime}\right) U^{\prime}\right\rangle-\left\langle\alpha U, \tilde{A} U^{\prime \prime}\right\rangle .
\end{aligned}
$$


Integrating the last term by parts and simplifying gives

$$
\begin{aligned}
\langle U .(\lambda & \left.+\bar{\lambda}+1) \alpha-s \alpha L R^{\prime}+s \alpha^{\prime} V\right\rangle+\left\langle s U^{\prime}-\lambda U, \alpha V\right\rangle \\
= & \left\langle U,\left(\alpha \Lambda L R^{\prime}-\alpha \tilde{A} L R^{\prime \prime}\right) U\right\rangle+\left\langle U,\left(\alpha \Lambda-2 \alpha \tilde{A} L R^{\prime}+(\alpha \widetilde{A})^{\prime}\right) U^{\prime}\right\rangle \\
& +\left\langle U^{\prime}, \alpha \tilde{A} U^{\prime}\right\rangle .
\end{aligned}
$$

By writing $V$ in terms of $U$ and its derivatives from (15a), we have

$$
\begin{aligned}
& \left\langle U,\left((2 \mathcal{R} e(\lambda)+1) \alpha-s\left(\alpha L R^{\prime}\right)^{*}-s \alpha L R^{\prime}+s \alpha^{\prime}\right)\left(s\left(U^{\prime}+L R^{\prime} U\right)-\lambda U\right)\right\rangle \\
& \quad+\langle V, \alpha V\rangle=\left\langle U,\left(\alpha \Lambda L R^{\prime}-\alpha \tilde{A} L R^{\prime \prime}\right) U\right\rangle \\
& \quad+\left\langle U,\left(\alpha \Lambda-2 \alpha \widetilde{A} L R^{\prime}+(\alpha \widetilde{A})^{\prime}\right) U^{\prime}\right\rangle+\left\langle U^{\prime} . \alpha \widetilde{A} U^{\prime}\right\rangle .
\end{aligned}
$$

Let

$$
\begin{aligned}
& E=-s \alpha L R^{\prime}-s\left(\alpha L R^{\prime}\right)^{*}+s \alpha^{\prime}, \\
& N=s E+2 \alpha \widetilde{A} L R^{\prime}-(\alpha \widetilde{A})^{\prime}, \\
& M=\left(\alpha \Lambda L R^{\prime}-\alpha \widetilde{A} L R^{\prime \prime}\right)+\lambda E-((2 \mathcal{R} e(\lambda)+1) \alpha+E) s L R^{\prime} .
\end{aligned}
$$

Then we have

$$
\begin{aligned}
& \left\langle U .((2 \mathcal{R e}(\lambda)+1) s \alpha-\alpha \Lambda) U^{\prime}\right\rangle+\left\langle U, N U^{\prime}\right\rangle+\langle V, \alpha V\rangle \\
& \quad=\lambda\left\langle U,\left(2 \mathcal{R} e^{e}(\lambda)+1\right) \alpha U\right\rangle+\left\langle U^{\prime}, \alpha \tilde{A} U^{\prime}\right\rangle+\langle U, M U\rangle .
\end{aligned}
$$

Take the real part:

$$
\begin{aligned}
& -\frac{1}{2}\left\langle U,\left((2 \mathcal{R e}(\lambda)+1) s \alpha^{\prime}-(\alpha \Lambda)^{\prime}\right) U\right\rangle+\mathcal{R} e\left\langle U, N U^{\prime}\right\rangle+\langle V, \alpha V\rangle \\
& =\operatorname{Re}(\lambda)(2 \mathcal{R} e(\lambda)+1)\langle U, \alpha U\rangle+\left\langle U^{\prime} \cdot \alpha \tilde{A} U^{\prime}\right\rangle+\mathcal{R} e\langle U, M U\rangle .
\end{aligned}
$$

In the weak shock limit, $N$ and $M$ are $O\left(\left|\widehat{U}_{x}\right|\right)$, while $N^{\prime}$ is $O\left(\left|\widehat{U}_{x x}\right|+\left|\widehat{U}_{x}\right|^{2}\right)$. Thus by Young's inequality, all the $\left|u_{1}\right|^{2}$ terms in $N$ can be made arbitrarily small. The $\left|\left(L R^{\prime}\right)_{11} \| u_{1}\right|^{2}$ terms vanish by (16). Thus, all the terms can be absorbed to give

$$
\begin{aligned}
& \sum_{j=1}^{N} \int_{-\infty}^{+\infty} \alpha_{j} \tilde{A}_{j}\left|u_{j}^{\prime}\right|^{2}+\frac{1}{2} \sum_{j=1}^{N} \int_{-\infty}^{+\infty}\left(s \alpha_{j}^{\prime}-\left(\alpha_{j} \Lambda_{j}\right)^{\prime}\right)\left|u_{j}\right|^{2} \\
& \quad \leq \sum_{j=1}^{N} \int_{-\infty}^{+\infty} \alpha_{j}\left|v_{j}\right|^{2}+C_{2} \int_{-\infty}^{+\infty}\left|\widehat{U}_{x}\right|\left[\epsilon_{2}\left|u_{1}\right|^{2}+\frac{1}{\epsilon_{2}} \sum_{j \neq 1}\left|u_{j}\right|^{2}+\sum_{j=1}^{N}\left|u_{j}^{\prime}\right|^{2}\right] .
\end{aligned}
$$

5. Remarks on Hypothesis (5). In our hypothesis, we assume that $A$ and $D f$ are simultaneously diagonalizable. It seems that a more natural, general, and interesting hypothesis would be to assume that (11) is symmetrizable; i.e., there exists some positive, symmetric multiplier $P=P(U, V)$ such that the following are symmetric:

$$
P \cdot\left(\begin{array}{ll}
0 & I \\
A & 0
\end{array}\right) \text { and } P \cdot\left(\begin{array}{cc}
0 & 0 \\
D f(\widehat{U}) & -I
\end{array}\right) \text {. }
$$

However the following proposition shows that these are equivalent assumptions. 
Proposition 1. Assuming hypothesis (5), the Jin-Xin model is symmetrizable if and only if $A$ and $D f$ are simultaneously diagonalizable, i.c., $[A, D f]=0$.

Proof. Let

$$
P=\left(\begin{array}{cc}
\alpha & \beta \\
\beta^{T} & \gamma
\end{array}\right)
$$

be a symmetrizer for (11), where $\alpha, \beta$, and $\gamma$ are $n \times n$ matrix-valued functions in $(U, V)$ and moreover $\alpha, \gamma$ are symmetric, positive-definite. Then left multiplying (11) by $P$ gives

$$
\begin{aligned}
\lambda P \cdot & \left(\begin{array}{l}
U \\
V
\end{array}\right)+\left(\begin{array}{cc}
\beta \cdot A-s \alpha & \alpha-s \beta \\
\gamma \cdot A-s \beta^{T} & \beta^{T}-s \gamma
\end{array}\right)\left(\begin{array}{l}
U \\
V
\end{array}\right)_{x} \\
& =\left(\begin{array}{cc}
-\beta \cdot D f & -\beta \\
\gamma \cdot D f & -\gamma
\end{array}\right)\left(\begin{array}{l}
U \\
V
\end{array}\right) .
\end{aligned}
$$

From the hypothesis it follows that $\beta, \beta \cdot A$, and $\beta \cdot D f$ must all be symmetric. In addition, $\alpha=\alpha^{T}=\gamma \cdot A$ and $\beta=\beta^{T}=-\gamma \cdot D f$. Hence $P$ can be written as

$$
P=\left(\begin{array}{cc}
\gamma \cdot A & -\gamma \cdot D f \\
-\gamma \cdot D f & \gamma
\end{array}\right)=\left(\begin{array}{cc}
\gamma & 0 \\
0 & \gamma
\end{array}\right)\left(\begin{array}{cc}
A & -D f \\
-D f & I
\end{array}\right)
$$

Since $\gamma$ is positive-definite, it follows that $\gamma$ is invertible. Moreover, we have $\beta \cdot A=$ $-\gamma \cdot D f \cdot A$ is symmetric and hence

$$
\gamma \cdot D f \cdot A=(\gamma \cdot D f \cdot A)^{T}=A^{T} \cdot D f^{T} \cdot \gamma=A^{T} \cdot \gamma \cdot D f=\gamma \cdot A \cdot D f
$$

Therefore, since $\gamma$ is invertible, commutation follows, $D f \cdot A=A \cdot D f$. We remark that by (18), every symmetrizer can be uniquely determined by $\gamma$.

The converse goes as follows: suppose that $[A, D F]=0$ in $(U, V)$. Then there exists a matrix-valued function $S=S(U, V)$ such that $S \cdot A \cdot S^{-1}$ and $S \cdot D f \cdot S^{-1}$ are both diagonal. Thus, we let $\gamma=S^{T} \cdot S$ and show that

$$
P=\left(\begin{array}{ll}
\gamma & 0 \\
0 & \gamma
\end{array}\right)\left(\begin{array}{cc}
A & -D f \\
-D f & I
\end{array}\right)
$$

is a symmetrizer for (11). Note that $\gamma>0$ and symmetric. Left multiplying (11) by $P$ gives

$$
\begin{aligned}
\lambda P \cdot & \left(\begin{array}{l}
U \\
V
\end{array}\right)+\left(\begin{array}{cc}
-s \gamma \cdot A-\gamma \cdot D f \cdot A & -\gamma \cdot A+s \gamma \cdot D f \\
-s \gamma \cdot D f-\gamma \cdot A & \gamma \cdot D f-s \gamma
\end{array}\right)\left(\begin{array}{l}
U \\
V
\end{array}\right)_{x} \\
& =\left(\begin{array}{cc}
-\gamma \cdot D f^{2} & \gamma \cdot D f \\
\gamma \cdot D f & -\gamma
\end{array}\right)\left(\begin{array}{l}
U \\
V
\end{array}\right) .
\end{aligned}
$$

Thus, it suffices to show that $\gamma \cdot A, \gamma \cdot D f, \gamma \cdot D f \cdot A$, and $\gamma \cdot D f^{2}$ are all symmetric and that $P$ is positive. Note that since $S \cdot A \cdot S^{-1}$ and $S \cdot D f \cdot S^{-1}$ are diagonal, it follows 
that $S \cdot A \cdot S^{-1}=\left(S^{-1}\right)^{T} \cdot A^{T} \cdot S^{T}$ and $S \cdot D f \cdot S^{-1}=\left(S^{-1}\right)^{T} \cdot D f^{T} \cdot S^{T}$. Thus,

$$
\begin{aligned}
(\gamma \cdot A)^{T} & =\left(S^{T} \cdot S \cdot A\right)^{T} \\
& =\left(S^{T} \cdot\left(S \cdot A \cdot S^{-1}\right) \cdot S\right)^{T} \\
& =\left(S^{T} \cdot\left(\left(S^{-1}\right)^{T} \cdot A^{T} \cdot S^{T}\right) \cdot S\right)^{T} \\
& =\left(A^{T} \cdot \gamma\right)^{T} \\
& =\gamma \cdot A .
\end{aligned}
$$

The others follow similarly. Hence, $P$ is symmetric and makes (19) symmetric as well. Finally, we show that $P$ is positive: Let

$$
y=\left(\begin{array}{ll}
S & 0 \\
0 & S
\end{array}\right) \cdot x
$$

Then

$$
\begin{aligned}
\langle x, P \cdot x\rangle & =\left\langle x,\left(\begin{array}{cc}
S^{T} S & 0 \\
0 & S^{T} S
\end{array}\right)\left(\begin{array}{cc}
A & -D f \\
-D f & I
\end{array}\right) \cdot x\right\rangle \\
& =\left\langle y \cdot\left(\begin{array}{cc}
S & 0 \\
0 & S
\end{array}\right)\left(\begin{array}{cc}
A & -D f \\
-D f & I
\end{array}\right)\left(\begin{array}{cc}
S^{-1} & 0 \\
0 & S^{-1}
\end{array}\right) \cdot y\right\rangle \\
& =\left\langle y \cdot\left(\begin{array}{cc}
\widetilde{A} & -\Lambda \\
-\Lambda & I
\end{array}\right) \cdot y\right\rangle .
\end{aligned}
$$

where $\widetilde{A}=S A S^{-1}$ and $\Lambda=S \Lambda S^{-1}$ are both diagonal. Thus, by reordering the coordinates of $y$, we can write the matrix as block diagonals of the form

$$
\left(\begin{array}{cc}
\tilde{A}_{i} & -\Lambda_{i} \\
-\Lambda_{i} & 1
\end{array}\right) .
$$

Hence, we have positivity if and only if each block is positive. However, a $2 \times 2$ block is positive if the trace and determinant are both positive, which follows from the fact that $\widetilde{A}$ is positive and condition (5), that $\widetilde{A}-\Lambda^{2}>0$.

Proposition 2. Assuming that the Jin-Xin model is symmetrizable, if $f$ is "genuinely coupled", in the sense that there are no constant invariant subspaces other than $\{0\}$ and $\mathbb{R}^{n}$, then $A=a I$, where $a$ is a scalar.

Proof. By the above proposition, symmetrizability implies that $A$ and $D f$ commute. Hence, because $D f$ is diagonalizable, $A$ is also. Moreover, every eigenspace of $A$ is an invariant subspace of $D f$. Thus, every eigenspace of $A$ is either $\{0\}$ or $\mathbb{R}^{n}$. Since the former is impossible, it follows that there is only one eigenspace, $\mathbb{R}^{n}$, and since $A$ is diagonalizable, it can be written as $A=a I$.

\section{REFERENCES}

[B] L. Q. Brin, Numerical testing of the stability of viscous shock waves, Math. Comp., 70 (2001). no. $235,1071-1088$

[Go] P. Godillon, Stabilité linéaire des profils pour les systémes avec relaxation semi-linéaire, Phys. D. 148 (2001), no. 3-4, 289-316 
[G] J. Goodman, Nonlinear asymptotic stability of viscous shock profiles for conservation laws, Arch. Rat. Mech. 95 (1986), 325-344

[HuZ] J. Humpherys and K. Zumbrun, Spectral stability of small-amplitude shock profiles for dissipative symmetric hyperbolic-parabolic systems, Z. Agnew. Math. Phys. 53 (2002), no. 1, 20-34

[JX] S. Jin and Z. P. Xin, The relaxation schemes for systems of conservation laws in arbitrary space dimensions, Comm. Pure Appl. Math. 48 (1995), no. 3, 235-276

[L] H. Liu, Asymptotic Stability of Relaxation Shock Profiles for Hyperbolic Conservation Laws, preprint

[MP] A. Majda and R. L. Pego, Stable viscosity matrices for systems of conservation laws, J. Diff. Eqs. 56 (1985), 229-262

[MZ] C. Mascia and K. Zumbrun, Pointwise Green's bounds and stability of relaxation shocks, in preparation

[Ze] Y. Zeng, Gas dynamics in thermal nonequilibrium and general hyperbolic systems with relaxation, Arch. Ration. Mech. Anal. 150 (1999), no. 3, 225-279

[Z] K. Zumbrun, Stability of multidimensional shock fronts, Lecture Notes, TMR Summer School, Köchel, May 1999

[ZH] K. Zumbrun and P. Howard, Pointwise semigroup methods and stability of viscous shock waves, Indiana Univ. Math. J. 47 (1998), no. 3, 741-871 